Supplementary Information

\title{
Positioning the Intracellular Salt Potassium Glutamate in the Hofmeister Series by Chemical Unfolding Studies of NTL9
}

\author{
Rituparna Sengupta ${ }^{1}$, Adrian Pantel ${ }^{2}$, Xian Cheng $^{1}$, Irina Shkel ${ }^{2,}{ }^{3}$, Ivan Peran ${ }^{4}$, Natalie Stenzoski ${ }^{4}$, \\ Daniel P. Raleigh ${ }^{4}$ and M. Thomas Record, Jr. ${ }^{1,2,3, *}$ \\ Program in Biophysics ${ }^{1}$ and Departments of Biochemistry ${ }^{2}$ and Chemistry ${ }^{3}$, University of Wisconsin-Madison, Madison, \\ WI 53706, USA \\ Department of Chemistry ${ }^{4}$, SUNY Stony Brook, Stony Brook, NY 11794-3400, USA
}

\section{GuHSCN Titrations}

To compare the effect of each $\mathrm{K}^{+}$- salt on NTL9 unfolding in GuHSCN, data was obtained at 1M total ionic concentration for each $\mathrm{K}^{+}-$salt (Fig 2). The data in Fig 2 as well as Fig 1A that shows GuHSCN unfolding in molar scale, was fit using an equation analogous to Eq. 8 in molar scale using $r_{4, M}=0$ ( since change in molar concentration of $\mathrm{K}^{+}-$salt, $d M_{4}=0$ ).

$$
F=F_{f}-\left(F_{f}-\left[F_{u}^{0}+S_{3} M_{3}\right]\right)\left(\frac{K_{o, M} e^{-\left(r_{3, M} M_{3}\right)}}{1+K_{o, M} e^{-\left(r_{3, M} M_{3}\right)}}\right) \quad \text { Eq. S1 }
$$

Here, $M_{3}$ is molar concentration of GuHSCN, $r_{3, M}$ is the $r$-value on the molar scale, and $K_{0, M}$ is the value of $K_{o}$ when evaluated in molar scale. This fit is used to compare unfolding in absence (Fig 1A) and presence of KGlu (Fig 1B) and also to compare between effects of each $\mathrm{K}^{+}-$salt on NTL9 unfolding. All $r$-values reported and analyzed in the text are on the molal scale (see Eqs. 1-7).

\section{Prediction of $\boldsymbol{r}$-values using $\alpha$-values and $K_{P}$ values from salt-model compound interaction data ${ }^{1}$}

$\alpha$-Values quantifying the interaction of a solute or salt with unit area of a given functional group or surface type are related to microscopic (local-bulk) partition coefficients $K_{P}$ and experimentally accessible $r$-values by the following equations.

$$
\begin{array}{cc}
\alpha_{i}=\frac{-v\left(K_{P, i}-1\right) b_{1}\left(1+\epsilon_{ \pm}\right)}{55.5} & \text { Eq. S2 } \\
r-\text { value }=\sum \alpha_{i} \times \Delta A S A_{i} & \text { Eq. S3 }
\end{array}
$$

Interaction potentials $\alpha_{i}$ are a measure of the free energy of interaction of the salt ions with unit surface area of a particular functional group (i); $v$ is the number of ions per formula unit of salt ( $v=2$ for 1-1 salts; $v=3$ for 2-1 salts); $K_{P, i}$ is the partition coefficient relating the average local concentration of salt ions in the vicinity of a particular functional group $i$ to the bulk salt concentration; $b_{1}$ is the amount of water in the local region per unit area of a functional group, deduced to be $0.18 \mathrm{H}_{2} \mathrm{O} / \mathrm{A}^{2}$ (i.e. 2 layers of water for various groups and surfaces $\left.{ }^{1}\right) ; 1+\epsilon_{ \pm}$is the non-ideality of the salt and $\triangle A S A_{i}$ is the change in water accessible surface area of that functional group in a process like NTL9 unfolding. 
Neglecting groups that make only small contributions to the $\triangle$ ASA of NTL9 unfolding, Eq. S3 is a sum of hydrocarbon and amide contributions.

$$
r-\text { value }=\alpha_{H} \Delta A S A_{H}+\alpha_{A} \Delta A S A_{A} \quad \text { Eq. S4 }
$$

Here, the subscript $H$ indicates hydrocarbon and $A$ indicates amide surface type.

\section{Determining partition coefficients $K_{P, N T L 9}^{\text {salt }}$ for interaction of salts with the surface of NTL9 exposed in unfolding}

The composition of the $\triangle$ ASA for unfolding NTL9 is $70 \%$ hydrocarbon and $30 \%$ amide. The overall $\mathrm{K}_{\mathrm{P}}$ can be written in terms of contribution from the hydrocarbon $\left(K_{P, H}\right)$ and amide $\left(K_{P, A}\right)$ surfaces for NTL9 unfolding.

$$
K_{P, N T L 9}^{\text {salt }}=0.7 K_{P, H}+0.3 K_{P, A} \quad \text { Eq. S5 }
$$

Contribution of hydrocarbon $\left(0.7 K_{P, H}\right)$ and amide $\left(0.3 K_{P, A}\right)$ towards the total $r$-value (Fig 6) was calculated using literature salt $K_{P}$ values for hydrocarbon and amide surface ${ }^{1}$.

Average $K_{P}$ values for interaction of KGlu with hydrocarbon and amide $\triangle$ ASA for unfolding NTL9 were calculated from KGlu $K_{P}$ values for interaction with the aliphatic and aromatic hydrocarbon (for NTL9, aliphatic C: aromatic $\mathrm{C}=7.3: 1$ ) and for interaction of KGlu with amide $\mathrm{O}$ and $\mathrm{N}$ (for NTL9, amide O: amide $\mathrm{N}=1.1: 1$ ) (X. Cheng, R. Sengupta and M. T. Record, Jr., unpublished observations) using Eqs. S6 and S7.

$$
\begin{array}{cc}
K_{P, \text { hydrocarbon }}^{K G \text { lu }}=\frac{7.3}{8.3} K_{P, \text { aliphatic } C}^{\text {KGlu }}+\frac{1}{8.3} K_{P, \text { aromatic } C}^{\text {KGlu }} & \text { Eq. S6 } \\
K_{P, \text { amide }}^{\text {KGlu }}=\frac{1.1}{2.1} K_{P, \text { amide } O}^{\text {KGlu }}+\frac{1}{2.1} K_{P, \text { amide } N}^{K G l u} & \text { Eq. S7 }
\end{array}
$$

The amide $\mathrm{K}_{\mathrm{P}}$ for all salts except KGlu were obtained from reference 1, where salt - amide data was obtained as an average for different amide O: amide $\mathrm{N}$ ASA ratios.

$\mathrm{K}_{\mathrm{P}}$ values for salts $\left(K_{P, M X}\right)$ are related to individual ion $\mathrm{K}_{\mathrm{P}}$ values as follows.

$$
v K_{P, M X}=v_{+} K_{P, M^{+}}+v_{-} K_{P, X^{-}} \quad \text { Eq. } \mathrm{S} 8
$$

Here, $v_{+}$and $v_{\text {- }}$ are the total number of cations and anions in the salt, respectively; $K_{P, M}{ }^{+}$and $K_{P, X}$ are the partition coefficients for the respective ions.

\section{Estimating binding constant for possible binding of sulfate to NTL9}

The effect of binding of sulfate to native NTL9 can be estimated by assuming that $r_{4}$ in Eq. 4 is the $\operatorname{sum}\left(r_{4, \text { total }}\right)$ of the effects from (i) preferential interaction (PI) of $\mathrm{K}_{2} \mathrm{SO}_{4}$ with the $\triangle \mathrm{ASA}$ for unfolding NTL9 $\left(r_{4, P I}\right)$ and (ii) the stabilizing effect of sulfate binding to folded NTL9 $\left(r_{4, \text { bind }}\right)$. 
$r_{4, \text { total }}=r_{4, P I}+r_{4, \text { bind }}$

Eq. S9

The effect from binding can be written in terms of the sulfate binding constant $\left(K_{4}\right)$ for binding of sulfate to native NTL9.

$r_{4, \text { bind }}=\frac{K_{4}}{1+K_{4}[4]}$

Eq. S10

In Eq. S10, [4] $\left([4]=m_{4}\right)$ is the concentration of sulfate. Using Eqs. S9-S10, Eq. 4 is modified as follows.

$d \ln K_{o b s}=-r_{3} d m_{3}-r_{4, P I} d m_{4}-\frac{K_{4}}{1+K_{4} m_{4}} d m_{4}$

Integrating Eq. S11 we get,

$K_{o b s}=\frac{K_{o} e^{-\left(r_{3} m_{3}+r_{4, P I} m_{4}\right)}}{1+K_{4} m_{4}}$

Eq. S12

Using this relation to fit the sulfate data with previously obtained $r$-value for GuHSCN $\left(r_{3}=-3.00 \mathrm{~m}^{-1}\right)$ and predicted $r$-value for $\mathrm{K}_{2} \mathrm{SO}_{4}\left(r_{4, P I}=3.13 \mathrm{~m}^{-1}\right)$, we obtained the binding constant for sulfate binding to NTL9 $\left(K_{4}=2.65 \mathrm{~m}^{-1}\right)$. 
Fig S1:

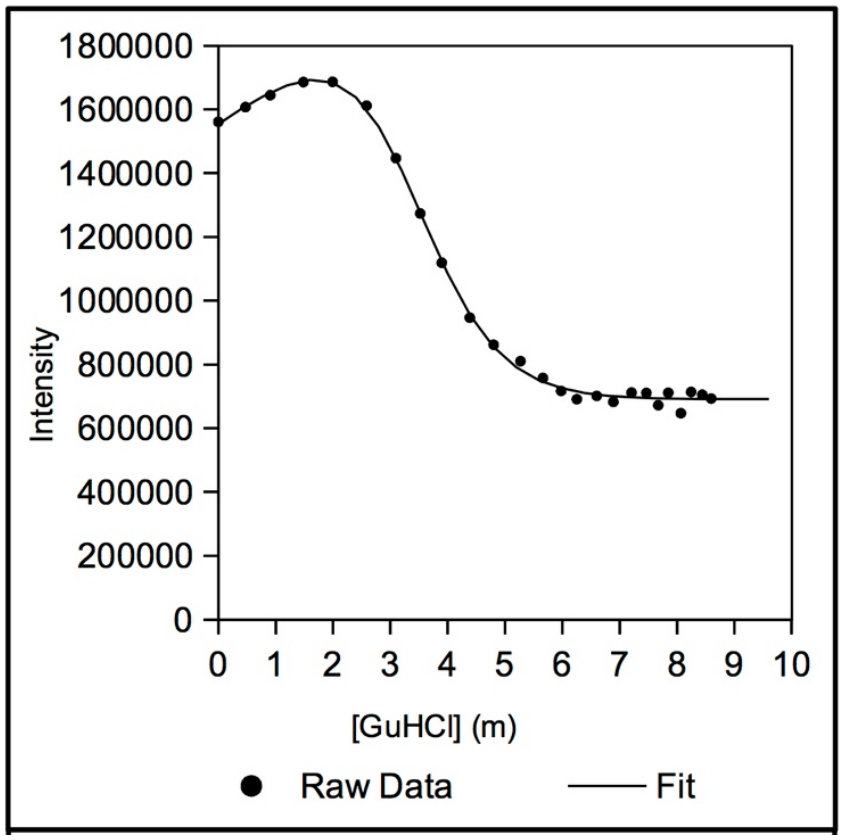

Fig S1: The plot shows Y25 fluorescence as a function of molal $\mathrm{GuHCl}$ concentration in NTL9 unfolding. The dots are raw data and the line represents the fit to the data (Eq. 9). Experiments were performed at $20^{\circ} \mathrm{C}$ in NB buffer.

Fig S2:

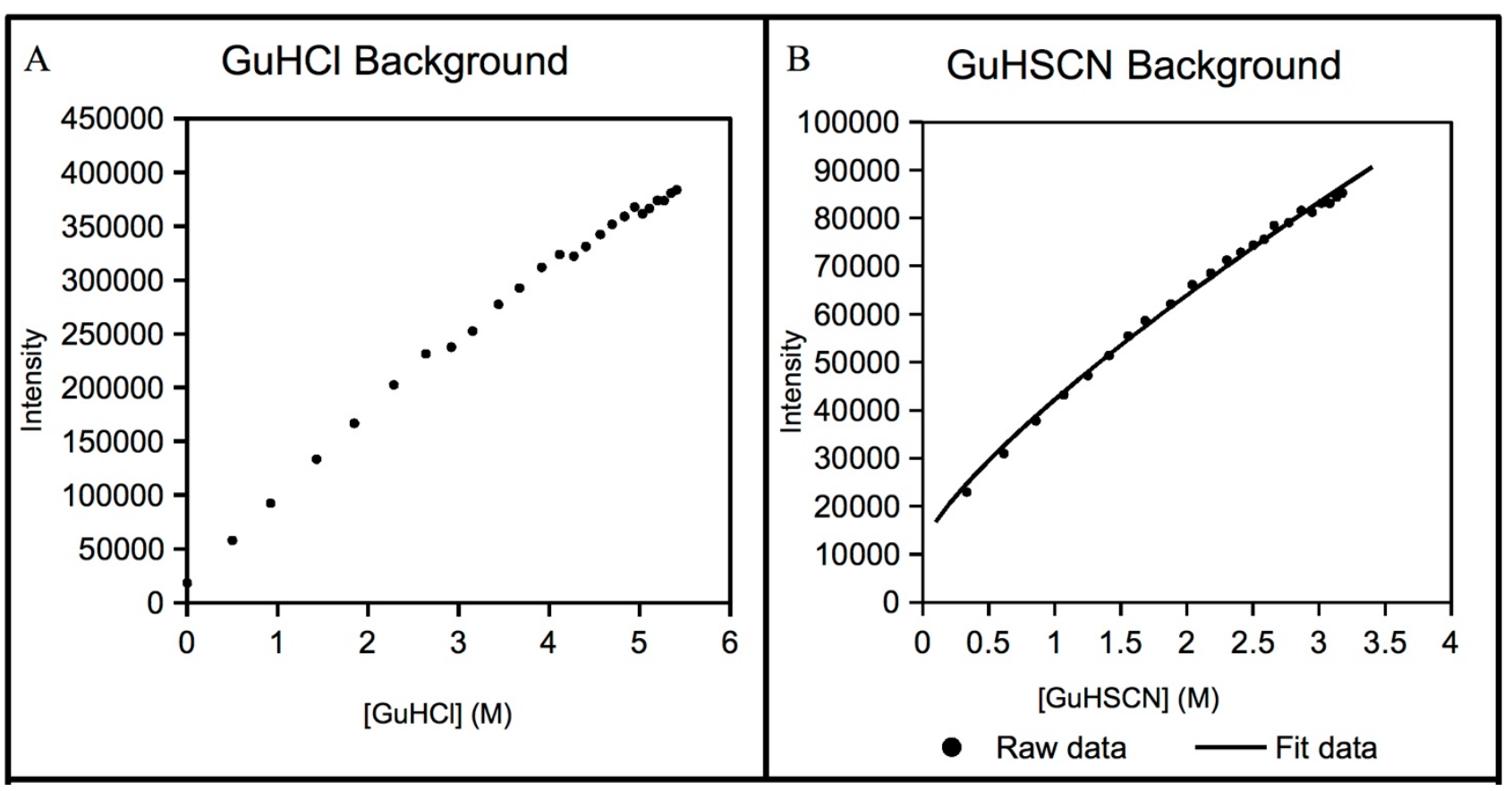

Fig S2: The plot shows (A) $\mathrm{GuHCl}$ and (B) GuHSCN background fluorescence at $303 \mathrm{~nm}$ as a function of molar $(\mathrm{M})$ concentration. The dots are raw data and the curve represents the fit to the GuHSCN data. Experiments were performed at $20^{\circ} \mathrm{C}$ in NB buffer. 
Fig S3:

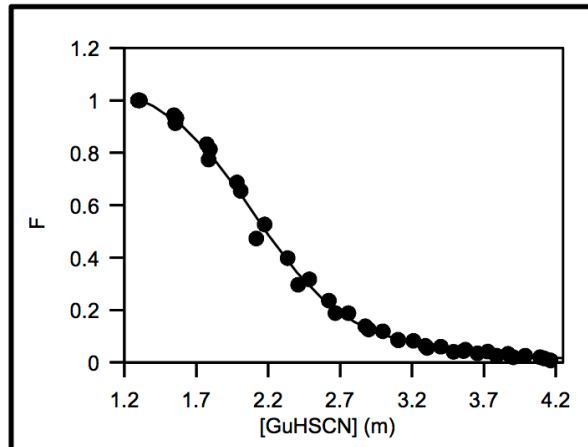

- Normalized Data $\quad$ Fit

Fig S3: Normalized fluorescence changes (F) of NTL9 in NB at $20^{\circ} \mathrm{C}$ as a function of GuHSCN concentration $(\mathrm{m})$ without added salt. The dots are data points and the line represents the fit to data.

Fig S4:

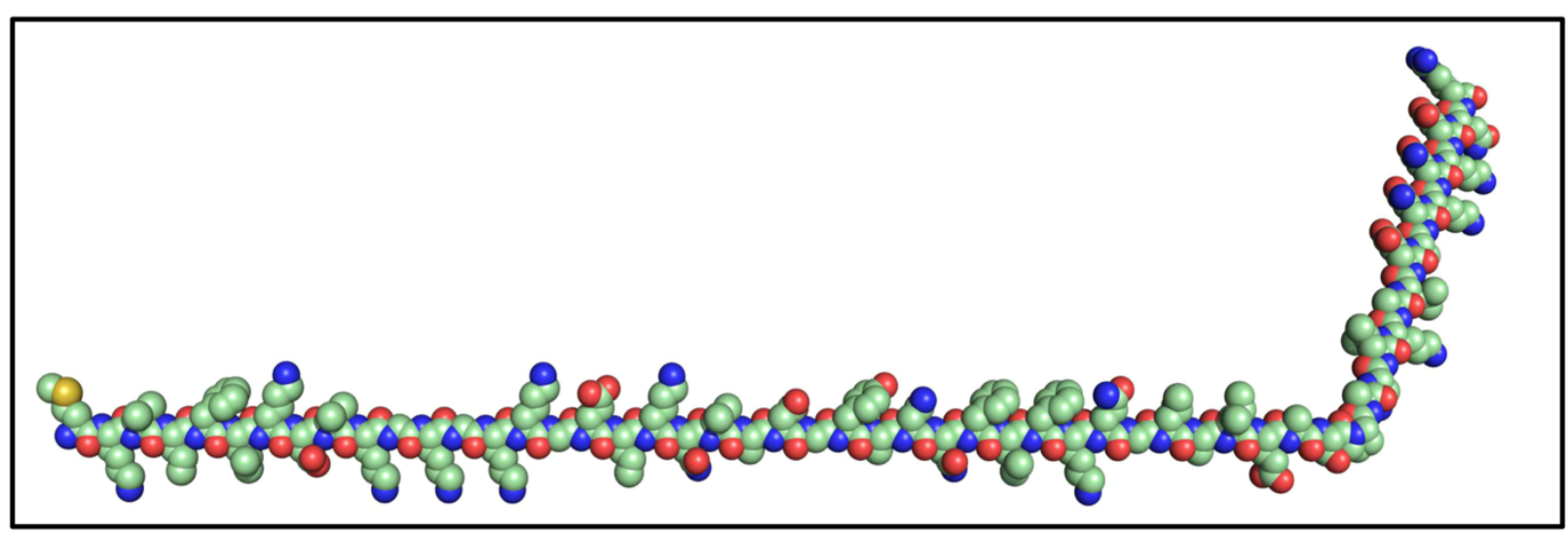

Fig S4: Model of maximally - extended, unfolded NTL9 used to calculate $\triangle \mathrm{ASA}_{\max }$.

Fig S5:
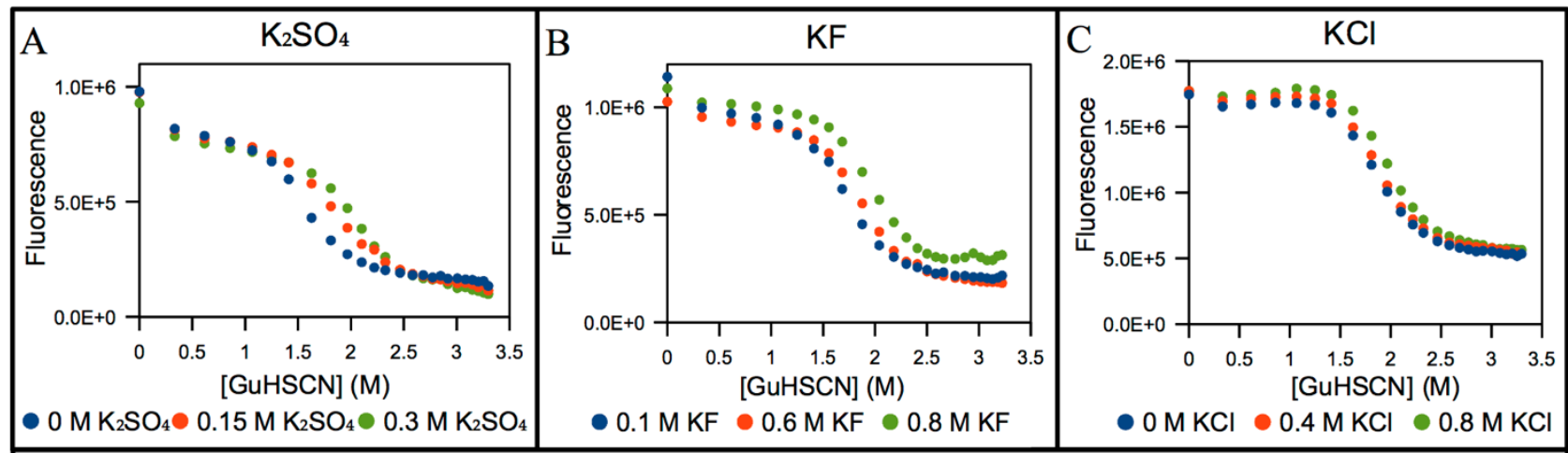

Fig S5: NTL9 fluorescence (background corrected) in NB at $20^{\circ} \mathrm{C}$ as a function of GuHSCN concentration at different molar concentrations of (A) $\mathrm{K}_{2} \mathrm{SO}_{4},(\mathrm{~B}) \mathrm{KF}$ and (C) $\mathrm{KCl}$. 
Table S1: Composition of $\triangle \mathrm{ASA}_{\max }$ and predicted $\triangle \mathrm{ASA}$ for NTL9 unfolding.

\begin{tabular}{|c|c|c|} 
Protein Functional Groups & $\Delta \mathrm{ASA}_{\max }$ & Predicted $\Delta \mathrm{ASA}$ \\
\hline Aliphatic $\mathrm{C}$ & $\mathbf{2 5 3 4}$ & $\mathbf{9 7 2}$ \\
\hline Aromatic C & $\mathbf{3 3 1}$ & $\mathbf{1 3 2}$ \\
\hline Amide $\mathrm{O}$ & $\mathbf{5 7 5}$ & $\mathbf{2 5 1}$ \\
\hline Amide N & $\mathbf{5 0 4}$ & $\mathbf{2 2 2}$ \\
\hline Carboxylate O & $\mathbf{1 4 6}$ & $\mathbf{0}$ \\
\hline Hydroxyl O & $\mathbf{5 4}$ & $\mathbf{0}$ \\
\hline Cationic N & $\mathbf{1 9 6}$ & $\mathbf{0}$ \\
\hline
\end{tabular}

Table S2: Summary of $K_{P}$ values of the salts for hydrocarbon and amide surface.

\begin{tabular}{|c|c|c|}
\hline & \multicolumn{2}{|c|}{${ }^{\mathrm{a}}$ Summary of $\mathrm{K}_{\mathrm{P}}$ values for: } \\
\hline Salt & Hydrocarbon & Amide \\
\hline GuHSCN & $\mathbf{1 . 3 5}$ & $\mathbf{1 . 4 5}$ \\
\hline $\mathrm{GuHCl}$ & $\mathbf{1 . 0 5}$ & $\mathbf{1 . 4 5}$ \\
\hline $\mathrm{KCl}$ & $\mathbf{0 . 6 5}$ & $\mathbf{1 . 6 0}$ \\
\hline $\mathrm{KF}$ & $\mathbf{0 . 5 0}$ & $\mathbf{1 . 5 0}$ \\
\hline $\mathrm{KGlu}$ & $\mathbf{0 . 6 0}$ & $\mathbf{0 . 9 1}$ \\
\hline $\mathrm{K}_{2} \mathrm{SO}_{4}$ & $\mathbf{0 . 0 7}$ & $\mathbf{2 . 1 3}$ \\
\hline
\end{tabular}

${ }^{\mathrm{a}} \mathrm{K}_{\mathrm{P}}$ values were obtained from reference 1 for everything except KGlu.

${ }^{\mathrm{b}}$ Obtained from or calculated (Eqs S6 - S7) using information from unpublished work of X. Cheng, R. Sengupta and M. T. Record, Jr.

Table S3: Summary of parameters used in calculations.

\begin{tabular}{|c|c|c|c|c|c|c|}
\hline Parameters & GuHSCN & $\mathrm{GuHCl}$ & $\mathrm{KCl}$ & $\mathrm{KF}$ & KGlu & $\mathrm{K}_{2} \mathrm{SO}_{4}$ \\
\hline$v$ & 2 & 2 & 2 & 2 & 2 & 3 \\
\hline $\mathrm{dOsm} / \mathrm{d} m_{2}{ }^{\mathrm{c}}$ & $1.63^{d}$ & 1.62 & 1.78 & 1.83 & $1.77^{\mathrm{e}}$ & 1.95 \\
\hline $1+\epsilon_{ \pm}=\left(\mathrm{dOsm} / \mathrm{d} m_{2}\right) / v$ & 0.815 & 0.81 & 0.89 & 0.915 & 0.885 & 0.65 \\
\hline$b_{1}{ }^{\mathrm{f}}$ & \multicolumn{6}{|c|}{$\mathbf{0 . 1 8}$ (for all salts) } \\
\hline
\end{tabular}

${ }^{c} \mathrm{dOsm} / \mathrm{d} m_{2}$ values obtained from reference 2 for everything except KGlu.

$\mathrm{d} \mathrm{dOsm} / \mathrm{d} m_{2}$ value for GuHSCN assumed to be similar to $\mathrm{GuHBr}$ (1.63) based on similarity between values for $\mathrm{NaSCN}$ and $\mathrm{NaBr}^{2}$.

${ }^{\mathrm{e}} \mathrm{dOsm} / \mathrm{d} m_{2}$ value for KGlu obtained from unpublished work of X. Cheng, R. Sengupta and M. T. Record, Jr.

${ }_{\mathrm{f}}$ Obtained from reference 1 . 


\section{REFERENCES}

1. Pegram L. M. and Record M. T. Jr. (2008) Thermodynamic origin of Hofmeister effects, J. Phys. Chem. B, 112: 9428 - 9436.

2. Pegram L. M. and Record M. T. Jr. (2007) Hofmeister salt effects on surface tension arise from partitioning of anions and cations between bulk water and air - water interface, J. Phys. Chem. B, 111: $5411-5417$. 